\section{Plasma concentrations of macrophage migration inhibitory factor are elevated in Pima Indians compared to Caucasians and are associated with insulin resistance}

To the Editor: Macrophage migration inhibitory factor (MIF) is thought to be a key effector molecule of both the innate and antigen-specific immune system [1]. While macrophages are the major source of MIF, MIF is also produced in pancreatic beta cells, adipocytes, liver, Leydig cells and ovarian follicular cells. When released in response to proinflammatory stimuli MIF inhibits random migration of macrophages and enhances macrophage functions and cytokine production [2]. Recently, it has been shown that MIF and insulin co-localize within the secretory granules of pancreatic beta cells [3]. Once released, MIF seems to regulate insulin secretion in an autocrine fashion [3], which suggests a potential role of MIF in the regulation of glucose metabolism, a hypothesis, which is supported by the finding of increased plasma MIF concentrations in diabetic patients [4]. We examined: (i) whether plasma MIF concentrations are related to insulin action or acute insulin secretory response in humans (ii) whether there are ethnic differences in plasma MIF concentrations between Caucasians and Pima Indians, a population at high risk for the development of Type II (non-insulin-dependent) diabetes mellitus [5].

The study group consisted of 28 non-diabetic (WHO) Pima Indians [age: $32 \pm 6$ years, body fat: $34 \pm 9 \%$ (DEXA or underwater weighing)] from the Gila River Indian Community near Phoenix, Arizona, USA and 24 non-diabetic Caucasian subjects [age: $30 \pm 7$ years, body fat: $33 \pm 7 \%$ ] who were admitted for 8 to 10 days to the NIH Clinical Research Unit in Phoenix, Arizona. All subjects were healthy with no acute or chronic infection. Blood samples were drawn after an overnight fast and $2 \mathrm{~h}$ after OGTT for measurements of plasma glucose, insulin and MIF concentrations (MIF- specific sandwich ELISA using recombinant human MIF as standard [6]).

There was no difference in age, percentage of body fat, fasting, and 2-h glucose concentrations, fasting and 2-h insulin

Received: 10 April 2002 / Accepted: 20 May 2002

Published online: 16 November 2002

(C) Springer-Verlag 2002 concentrations, and acute insulin response (AIR 25-g intravenous glucose tolerance test) between Pima Indians and Caucasians (data not shown). Insulin action ( $\mathrm{M}$, hyperinsulinaemic euglycaemic clamp [7]) was lower in Pima Indians compared to Caucasians $\left(3.75 \pm 1.18\right.$ and $4.72 \pm 1.79 \mathrm{mg} \cdot \mathrm{kgEMBS}^{-1} \cdot \mathrm{min}^{-1}$, respectively, $p<0.05)$. While in these healthy subjects, MIF was frequently below the detection level of the assay, the frequency distribution of MIF in Pima Indians was different from Caucasians (Fig. 1A) with higher fasting MIF concentrations in Pima Indians (Wilcoxon Rank sum test $p=0.04$ ). Thus fasting MIF concentrations were higher than $5 \mathrm{ng} / \mathrm{ml}$ in $39 \%$ of Pima Indians, but not in any of the Caucasians. Fasting MIF was below the level of detection of the assay in $71 \%$ of Caucasians, 54\% of Pima Indians (Fig. 1A). MIF concentrations at 2-h tended to be higher in Pima Indians compared to Caucasians (Wilcoxon Rank sum test $p=0.1$ ). There was no difference between fasting and 2-h MIF concentrations $(p=0.2)$. Fasting MIF concentrations were negatively associated with $\mathrm{M}$ before (Fig. 1B) and after adjustment for age, sex and percentage of body fat $(r=-0.80, p=0.0001)$. Moreover, those subjects who had undetectable fasting MIF concentrations had on average higher $\mathrm{M}$ values compared to those who had detectable fasting MIF concentrations $\left(4.7 \pm 1.5\right.$ and $3.1 \pm 0.7 \mathrm{mg} \cdot \mathrm{kgEMBS}^{-1} \cdot \mathrm{min}^{-1}$, respectively, $p=0.0005)$. 2-h MIF concentrations were not correlated with $\mathrm{M}(r=-0.06, p=0.9)$. There was no correlation between fasting or 2-h MIF and measures of adiposity (body fat and BMI), fasting and 2-h glucose and insulin and AIR before or after adjustment for age and sex (all $p>0.05$ ).

Another study [4] showed that serum MIF concentrations were higher in patients with Type II diabetes compared to their age and sex matched normal healthy control subjects. We extend this observation by showing higher MIF concentrations in non-diabetic Pima Indians, a population at high risk for the development of Type II diabetes, compared to non-diabetic Caucasians. A study carried out in isolated rat islets showed that MIF production is regulated by glucose [3]. Despite this, we show that MIF concentrations are not associated with fasting glucose, a finding in agreement with a previous study [4], and additionally find no association between serum concentrations of MIF and $2 \mathrm{~h}$ plasma glucose. MIF has also been proposed to act in a paracrine fashion to modulate insulin secretion. Thus, in vitro immunoneutralization of MIF was shown to reduce insulin secretion [3]. However, we show that MIF concentrations are not associated with fasting or 2-h insulin and acute insulin secretory response. By contrast, we show that fasting MIF concentrations are strongly associated with insulin resistance in vivo. Associations of insulin resistance and MIF in healthy human subjects have not been reported, although MIF is released 
A
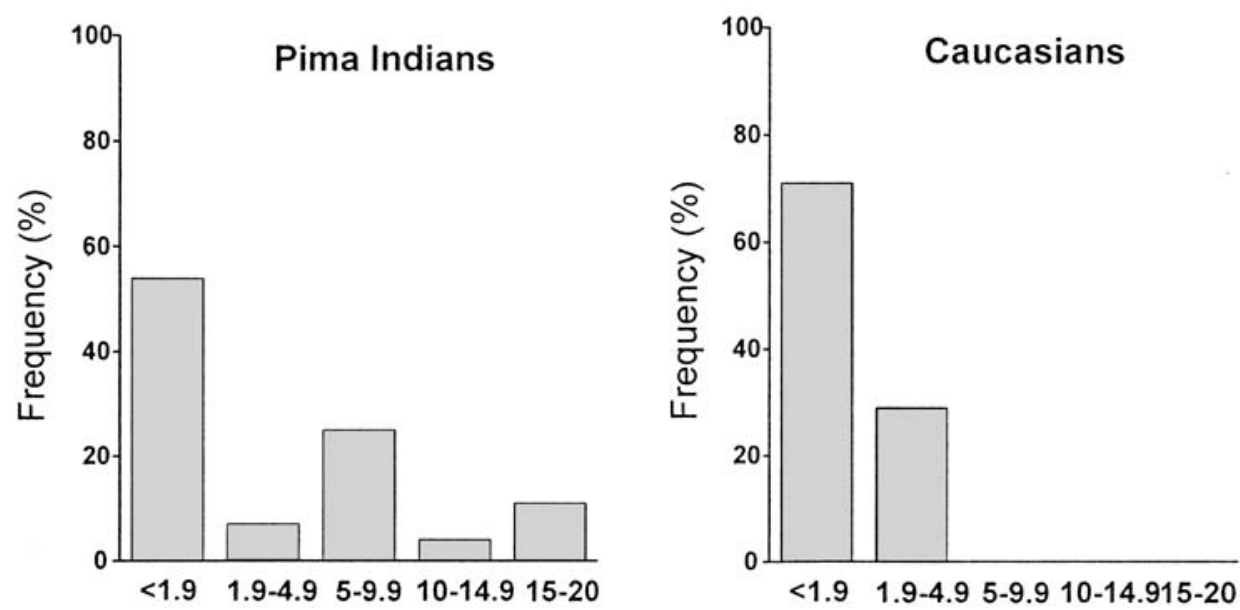

Fasting MIF concentrations ( $\mathrm{ng} / \mathrm{ml}$ )

Fasting MIF concentrations (ng/ml)

\section{B}

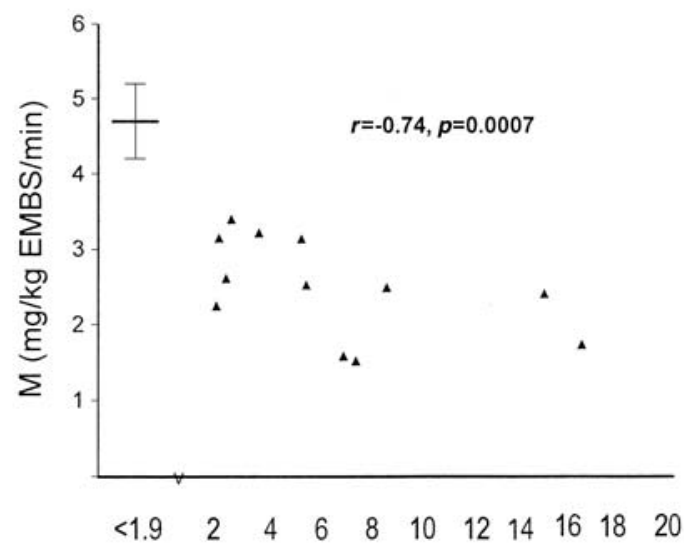

Fasting MIF concentrations ( $\mathrm{ng} / \mathrm{ml}$ )

Fig. 1. A Distribution of fasting plasma macrophage migration inhibitory factor concentrations (MIF) in Pima Indians and Caucasians. B Relationship between the fasting plasma macrophage migration inhibitory factor concentrations (MIF) and insulin action (M) in 36 non-diabetic Pima Indians and Caucasians. Subjects were divided into those with undetectable MIF levels (mean $\pm \mathrm{SE}$ ) and those with detectable levels. The detection limit of this MIF ELISA using plasma is between 1-2 ng/ml. Detection limit for this study was $1.9 \mathrm{ng} / \mathrm{ml}$. Many individuals had MIF levels that were below the detectable limit $(<1.9 \mathrm{ng} / \mathrm{ml})$. To account for this "truncation", the data were analysed using a Tobit model. This model assumes that MIF concentrations have log-normal distribution, but accounts for the fact that for some individuals the value is not known exactly, but is between 0 and $1.9 \mathrm{ng} / \mathrm{ml}$. A Fasting MIF concentrations (ng/ml). B Fasting MIF concentrations (ng/ml)

in disease states also associated with insulin resistance [8]. MIF was found to induce TNF-a secretion by macrophages, which suggests that MIF is not only increased by but also increases cytokine production [2]. Thus, MIF might have both direct and indirect effects on insulin sensitivity.
One limitation of our study is that, using currently available assays, MIF concentrations in healthy subjects were below the detection level of the assay, as was the case in $50 \%$ of subjects. Concentrations in the present study are typical of normal values in other studies, as is the limit of detection of MIF of the assay used (C. Metz, personal communication).

In conclusion, these results indicate that plasma MIF concentrations were increased in non-diabetic Pima Indians, a population at high risk for the development of the disease, compared to age and body composition matched Caucasian subjects and were strongly related to insulin resistance. Our results are consistent with the hypothesis that chronic inflammatory processes could play a role in pathogenesis of Type II diabetes.

Acknowledgements. The authors wish to thank members of the Gila River Indian Community for their participation. We also gratefully acknowledge the nurses of the Clinical Research Unit, as well as the staff of the metabolic kitchen for their care of the patients in these studies.

B. Vozarova, N. Stefan, R. Hanson, R.S. Lindsay, C. Bogardus, P.A. Tataranni, C. Metz, R. Bucala 


\section{References}

1. Bloom BR, Bennett B (1966) Mechanism of a reaction in vitro associated with delayed-type hypersensitivity. Science 153:80-82

2. Calandra T, Bernhagen J, Mitchell RA, Bucala R (1994) The macrophage is an important and previously unrecognized source of macrophage migration inhibitory factor. J Exp Med 179:1895-1902

3. Waeber G, Calandra T, Roduit R et al. (1997) Insulin secretion is regulated by the glucose-dependent production of islet beta cell macrophage migration inhibitory factor. Proc Natl Acad Sci USA 94:4782-4787

4. Yabunaka N, Nishihira J, Mizue Y et al. (2000) Elevated serum content of macrophage migration inhibitory factor in patients with type 2 diabetes. Diabetes Care 23:256-258

5. Knowler WC, Pettitt DJ, Saad MF, Bennett PH (1990) Diabetes mellitus in the Pima Indians: incidence, risk factors and pathogenesis. Diabetes Metab Rev 6:1-27
6. Calandra T, Echtenacher B, Roy DL et al. (2000) Protection from septic shock by neutralization of macrophage migration inhibitory factor. Nat Med 6:164-170

7. Lillioja S, Mott DM, Howard BV et al. (1988) Impaired glucose tolerance as a disorder of insulin action. Longitudinal and cross-sectional studies in Pima Indians. N Engl J Med 318:1217-1225

8. Calandra T, Bucala R (1995) Macrophage migration inhibitory factor: a counter-regulator of glucocorticoid action and critical mediator of septic shock. J Inflamm 47:39-51

Corresponding author: Dr. B. Vozarova, Clinical Diabetes and Nutrition Section, National Institutes of Health, 4212 N 16th Street. Rm. 5-41, Phoenix, AZ 85016, USA. E-mail: bvozarov@mail.nih.gov

Abbreviations: MIF, Macrophage migration inhibitory factor; M, insulin-stimulated glucose disposal during low dose insulin infusion; EMBS, estimated metabolic body size (fat-free mass $+17.7 \mathrm{~kg}$ ); AIR, acute insulin response 